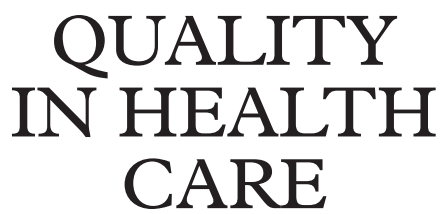

\title{
Editorials
}

\section{Decision analysis and the evaluation of decision technologies}

Most people have preferred ways of making judgements and decisions. When lost and not knowing what to do, some of us prefer to rely on our instinct, some on our experience based judgement. Some of us are prepared to ask our companions, some will prefer to approach people who are judged to be experts (police, newspaper vendors), and some would rather immediately consult a guide map, compass or other more analysis and evidence based guidance system. However, wherever we prefer to operate on the intuition analysis continuum, ${ }^{1}$ few of us are inclined to monitor the success of our preferred method, although our near and dear may do so and become frustrated by our obstinate refusal to change to theirs. Even fewer would be prepared to carry out an intervention based evaluation or even a less robust observational study of outcomes. So our progress around unfamiliar places is erratic and variable. However, at least it is rarely a matter of life and death and many will justify it as charming irrationality that can have serendipitous results.

Two papers published recently in Quality in Health Care - one by Timmermans and colleagues in this issue ${ }^{2}$ and the other by Robinson and Thomson on behalf of the Decision Analysis in Routine Treatments Study (DARTS) team $^{3}$ which appeared in the December issue - suggest that moving towards more analytical judgement and decision making will have considerable benefits in the more serious context of medical care, particularly in the growing number of situations where alternative management strategies have significantly different consequences in terms of quantity and quality of life, or different aspects of health related quality of life. It has been-and still is in many places - traditional for the necessary value judgments to be made implicitly by clinicians without any significant attempt to establish the preferences of the individual patient or, indeed, to address the trade offs explicitly themselves without involving the patient. With increased calls for patient empowerment, the issue of whose preferences are to be used in medical decisions, and how, is coming to the forefront and greater analysis is a necessary prerequisite of any attempt to integrate evidence based and preference driven care. ${ }^{4}$

However, the case for deeper analysis - that is, a higher analysis-to-intuition ratio in terms of Hammond's cognitive continuum - may rest simply on the complexity of calculating the consequences of the alternative options. Even using the apparently straightforward measure of life expectancy, the task of quantitatively modelling alternative scenarios at a patient specific level is beyond intuitive judgement, at least if the results of following correct statistical procedures are regarded as valid. There is a good example of this latter possibility in the study by Timmermans et al where it seems that the surgeons studied failed to realise that, for a particular group of patients (young people with smaller aneurysms), the cumulative risk of rupture in the long term is higher than the operative mortality risk.

Timmermans and colleagues compared the expressed decisions of four surgeons on 137 cases of patients with asymptomatic abdominal aortic aneurysms with the output of a decision analytical model for the treatment decision"elective surgery" or "watchful waiting". (They were "paper patients" but were based on real cases.) Using life expectancy (LE) and a quality adjusted life expectancy (QALE) measure as alternative maximands for the model, the researchers established the extent and nature of agreement between clinicians and the model. Agreement was fairly high with $81 \%$ (LE model) and $76 \%$ (QALE model) of the decisions being the same. Interestingly, where disagreement occurred, it was usually because the surgeons were less aggressive than the LE model but more aggressive than the QALE model. The details of patient characteristics associated with these differences, while interesting, are less important than the fact that they exist. Their existence confirms the need for increased analysis if we are to understand why decisions are made, why they differ, and how they can be improved.

Timmermans et al report, but do not stress, the difference between the surgeons. Their results are based on a comparison of the clinician average with the model, but it is obvious that variation between clinicians may be a major problem and the study supports the view that evaluations should really be of particular decision analytical models (with specified inputs) and individual clinicians. The idea that one can come to meaningful and relevant conclusions about the relative merits of "decision analysis" in the abstract with regard to "clinical judgement/intuition" in general is absurd.

If quality adjustments are as vital as they can be seen to be in the study by Timmermans et $a l,{ }^{2}$ they too need to be addressed much more analytically. Robinson and Thom$\operatorname{son}^{3}$ do so, concentrating on presenting the expected utility theory that underlies their decision analysis based decision aid. The DARTS study was designed to enable patients to make a "shared" and "informed" decision about whether or not to take warfarin for their atrial fibrillation. It involved an elicitation phase in which the patient's utilities were established in relation to five disease specific states, an 
informational phase in which selected evidence based and risk factor adjusted probabilities for these were presented to him or her, and an end phase in which the patient, having told the programme about his/her current intention, was informed whether or not it concurred. They then entered into final decision making dialogue with the doctor who had been delivering the programme from the beginning. At no point were the actual numerical results of the expected utility calculations for the two options underlying the model's agreement or disagreement (based on the elicitation of phase 1 and the probabilities underlying phase 2) made available. This distinguishes the approach of the DARTS team from similar instantiations of decision analysis based approaches which expose the full calculations in the interests of transparency, as well as permitting "what if" explorations of sensitivity. The Stanford approach to dynamic and distributed decision making in the form of PORTAL and ALCHEMIST ${ }^{5}$ and our own CODA programme adopt this position, though the latter is designed to allow the depth of analysis and extent of quantification to be varied by the users. ${ }^{6}$

The design of the study by Timmermans et al did not allow for any involvement or communication with the patient, whereas in the study reported by Robinson and Thomson it was the central, if not the only, motivation for the study. These two papers therefore show that decision analysis can be used in a variety of ways and with varying intentions. It can be put into the service of any variant of the doctor-patient relationship, insofar as it essentially raises the analytical level of each component of the judgement-decision making process. But once this is realised and accepted, it becomes clear that we need to distinguish the preference of each party (doctor and patient) regarding the nature of their relationship from the preferred mode of judgement and decision making of each within that preferred form of relationship. The two are no longer mutually determined as they were, and are, in traditional clinical judgement. For example, it becomes entirely sensible for a patient to say "Do what is best for me, doctor. I don't want to be informed to any significant extent (because I know that even simplest Markov modelling of most choices shows that being 'fully' informed is a nonsensical goal), but I do want you to determine what is best for me by consulting a decision analytical model personalised to my risk factors which is able to incorporate my utilities".

Evaluation of decision aids and support systems will accordingly need to be undertaken in the light of these "meta-preferences" of patient and doctor regarding relationship type and analytical mode; otherwise, our evaluations will merely reflect them or be heavily confounded by them. In the light of the results of the study by Timmermans et al it also becomes clear that we must be very wary of taking the output from an individual operating at one mode (even the supposedly best individual) and regarding that as the gold standard. As more and more implementations of analytical decision technologies come on stream, it will increasingly be a question of deciding how far the views of those with heavy investment in the existing technology are allowed to determine (through the use of evaluation dimensions such as "acceptability") the rate at which the new alternatives are developed and implemented. The evaluation of decision technologies must surely be carried out in the same way as the evaluation of pharmaceutical, imaging, and other medical technologies - that is, highly analytically. Even if this will be very difficult-and some clinicians will argue that it is unethical, unlawful, or both-there can be no normative justification for doing anything else. If it is currently unlawful by the standard "reasonable practitioner" tests, we will simply have to conclude that, until the community accepts that all instantiations of all decision technologies require serious and proper (comparative) evaluation, we-or, rather, patients - will have to pay the price potentially involved. The usefulness and feasibility in shared decision making of the model used by Timmermans et al is currently being evaluated in a prospective multicentre study and we await the results with interest.

JACK DOWIE

Professor of Health Impact Analysis, Public Health and Policy Department, London School of Hygiene and Tropical Medicine, London WC1E 7HT, UK

fack.Dowie@lshtm.ac.uk

1 Hammond KR. Human judgment and social policy: irreducible uncertainty, inevitable error, unavoidable injustice. New York: Oxford University Press; 1996.

2 Timmermans D, Kievit J, Bockel H. Improving the quality of surgeons' treatment decisions: a comparison of clinical decision making with a computerised evidence-based decision analytic model. Quality in Health Care 2001;10:4-9.

3 Robinson A, Thomson R G. The potential use of decision analysis to support shared decision making in the case of uncertainty: the example of atrial fibrillation and warfarin anticoagulation. Quality in Health Care 2000; 9:238-45.

4 Dowie J. 'Evidence based', 'cost-effective' and 'preference-driven' medicine: decision analysis based medical decision making is the pre-requisite. $\mathcal{F}$ Health Serv Res Policy 1996;1:104-13.

5 Sanders GD, Hagerty CG, Sonnenberg FA, et al. Distributed decision support using a web-based interface: prevention of sudden cardiac death. Med Decis Making 1999;19:157-66.

6 Pell I, Dowie J, Clarke A, et al. A Clinical guidance program for the incidental prophylactic oophorectomy decision. BMf (submitted for publication). 